\title{
Prebreakdown Conduction in Vacuum Gaps Under Switching IMPULSE Excitations
}

\author{
K. V. Srinivasa and G. R. Nagabhushana \\ Indian Institute of Science \\ Department of High Voltage Engineering \\ Bangalore, India
}

\begin{abstract}
The present investigation concerns prebreakdown conduction in vacuum gaps under slow impulses. Hemispherical, plane, and needle electrodes have been used in a vacuum environment of better than $0.1 \mathrm{mPa}$. The gap spacings were $0.5,1$, and $1.5 \mathrm{~mm}$. The front times ranged from $200 \mu \mathrm{s}$ to $5 \mathrm{~ms}$. The results indicate that prebreakdown currents were smaller than the highest sensitivity of $10^{-7}$ A for front times of about 1 ms. For larger front times, breakdown currents were observed, which increased with front time. The voltagecurrent relationship appears to follow Fowler-Nordheim theory but Fowler-Nordheim plots are branched. The high magnitudes may be attributed to ion-assisted field emission based on which a model has been developed, that agrees substantially with experimental results. Applicability of non-metallic type of emission is discussed.
\end{abstract}

\section{INTRODUCTION}

Prebreakdown conduction in vacuum gaps have been extensively studied under dc excitation, under clean conditions of vacuum and electrode surfaces. Though it is generally believed that Fowler-Nordheim [1] (F-N) theory satisfactorily explains the process of prebreakdown (pbd) conduction, in the recent past Latham and co-workers [2] Hurley et a1. [3] have shown that emission could be of the "non-metallic" type. The nonmetallic type of emission also gives rise to an approximately linear F-N plot having a high field intensification factor $\beta$. Very few studies have been reported on pbd conduction in vacuum gaps under ac and switching impulse excitations [4-11]. These studies are more complicated because of the presence of gap displacement current which is dominant at low applied voltages. In the present work pbd currents have been measured under slow impulses for different front times, gap settings, and electrode configurations.

\section{EXPERIMENTAL DETAILS}

The experiments were carried out in a vacuum better than $0.1 \mathrm{mPa}$. A liquid nitrogen trap was used to produce clean vacuum. The following electrode configurations have been scudied: (1) Hemisphere - plane with hemispheres of diameters of $3,10,20$, and $30 \mathrm{~mm}$ and plane electrodes of $30 \mathrm{~mm}$ diameter (edge rounded off to a radius of $1.5 \mathrm{~mm}$ ), (2) needle-plane, needle tip diameter $0.1 \mathrm{~mm}$ and plane as anode and (3) needle-needle. In each case, the first mentioned is the low-voltage (LV) electrode. Gap spacings of $0.5,1$, and $1.5 \mathrm{~mm}$ have been studied. The hemispheres and plane electrodes were of stainless stee1 Type 304 . Needles of stainless stee1 304 and also of a special alloy steel were used. The special alloy steel needles get mildly rusted when kept immersed in water over a day. These needles were used to study the effect of such rusting on pbd currents. The electrodes were polished to mirror finish with micron-sized alumina powder and ultrasonically cleaned before use. Great care was taken to prevent any contamination during handling. Impulse voltages were generated using a 4-stage Marx circuit $(50 \mathrm{kV}, 0.5 \mu \mathrm{F} /$ stage). A non-inductive resistor of $30 \mathrm{k} \Omega$ was used for limiting the current and minimizing the electrode damage on breakdown of the gap. A current sensing resistor of $0.81 \mathrm{k} \Omega$ was used in series with the LV electrode. The voltage and current were recorded with an oscilloscope. The front times of the impulses were $200 \mu \mathrm{s}, 750 \mu \mathrm{s}$, $1.7 \mathrm{~ms}, 3 \mathrm{~ms}$ and $5 \mathrm{~ms}$, with a common tail time of $15 \mathrm{~ms}$. Gap spacings of $0.5,1$, and $1.5 \mathrm{~mm}$ were studied. The electrodes were subjected to ac conditioning prior to the application of the impulses. This consisted of applying $50 \mathrm{~Hz}$ ac voltage at a low level and increasing slowly until a few sparks occurred, the current being limited to less than $0.5 \mathrm{~A}$ (peak). The voltage was then 
reduced to $80 \%$ of the sparking value and maintained constant for 10 minutes. From experimental observations, the maintenance voltages during ac conditioning were found to be 18,36 , and $48 \mathrm{kV}$ for $0.5,1$ and $1.5 \mathrm{~mm}$ gaps, respectively. A few experiments were also conducted with electrodes subjected to "spark conditioning" in which the gaps were subjected to 20 to 30 sparks with the current on sparkover being less than $0.5 \mathrm{~A}$ (peak).

The experiment started with the application of impulses at a low level and increasing in small steps. Five impulses were applied at each level at intervals of $30 \mathrm{~s}$. That voltage level at which there were at least two breakdowns (resulting in chopped waves as seen on the (RO) was defined to be the breakdown voltage.

The pbd current was obtained by subtracting the gap displacement current (corresponding to the applied voltages) from the total current. The highest sensitivity of current measurement was $10^{-7} \mathrm{~A}$.

\section{RESULTS AND DISCUSSIONS}

Prebreakdown currents have been measured for each of the above mentioned configurations. Additionally, some experiments were conducted to study the effect of contamination. (1) in one set of experiments, liquid nitrogen was not used in the trap so that back-streaming of diffusion pump fluid would contaminate the electrodes. This is designated "contaminated condition A", and (2) in another set of experiments, the special alloy needle, allowed to rust in water over a day, was used. This is designated "contaminated condition "B".

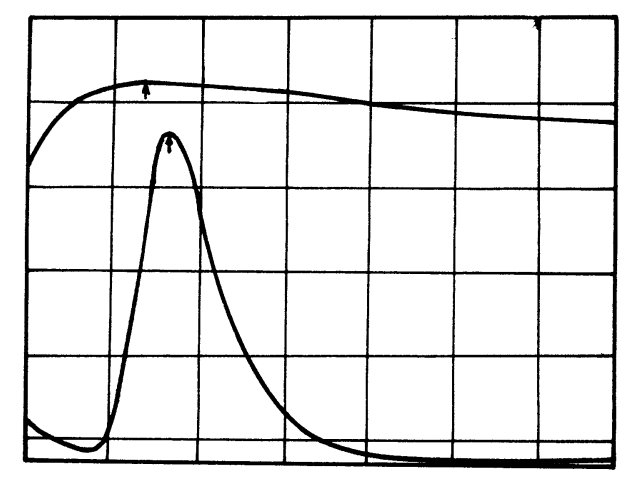

Fig. 1(a): Typical pbd current oscillogram of $3 \mathrm{~ms}$ Front impulse excitation

Upper beam (voltage) $120 \mathrm{kV} / \mathrm{div}$, Lower beam (current) $63 \mu \mathrm{A} / \mathrm{div}$,

Sweep $2 \mathrm{~ms} / \mathrm{div}$.

Peak has been indicated by an arrow.

In every case, the pbd current waveform was "asymmetric" in the sense that the current peak always occurred later than the voltage peak, the time lag ranging from 0.3 to $0.5 \mathrm{~ms}$. The current magnitude in the tail was higher for a given voltage. Fig. 1(a) shows a typical oscillogram. Fig. 1(b) shows time lag between the voltage and current peaks as a function of front time of the applied impulse. The consistent increase in the time lag with front time confirms that the time lag is due to phenomena in the gap. It has also been verified that the time lag due to the measuring circuit is $<1 \mu \mathrm{s}$, and therefore should be precluded. Similar time lags between voltage and current peaks and asymmetry in the current waveform have been observed also by earlier workers $[6,7,8,9]$. In addition to the delay and asymmetry, the pbd currents under impulse voltages have been found to be at least an order higher than under comparable $50 \mathrm{~Hz}$ ac stress. It is worth noting that pbd current magnitudes under $50 \mathrm{~Hz}$ voltages are about two orders higher than under dc voltages [7].

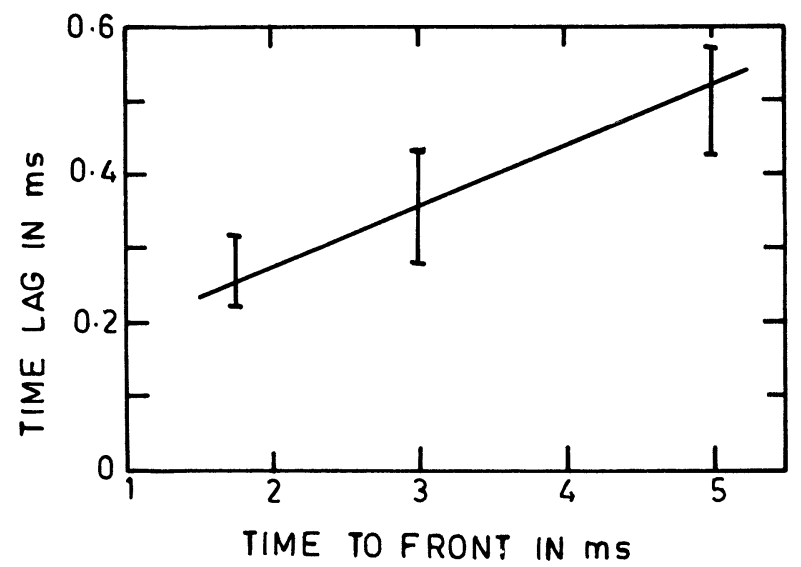

Fig. 1(b): Variation of time-lag between voltage and current peaks as a function of time to front of applied impulse voltages

\section{INFLUENCE OF TIME TO FRONT ON PBD CONDUCTION}

For any given gap configuration, pbd currents were below perceptible levels (i.e. $<<10^{-7} \mathrm{~A}$ ) for front times less than or equal to $750 \mu \mathrm{s}$. For $1.7 \mathrm{~ms}$ fronted impulses, a well-defined pbd current waveform developed occasionally. Over the regime of 200 to $750 \mu \mathrm{s}$ front times, microdischarges were observed frequently. These were typically rectangular pulses, magnttudes ranging to a few tens of $\mathrm{mA}$ and duration being around 1 to $2 \mathrm{~ms}$. Sometimes, a micro-discharge resulted in subsequent development of a well-defined pbd waveform and at some other times a pbd current culminated in a microdischarge. It was observed that pbd currents would be conditioned off with repeated application of impulses or by application of a higher voltage impulse. For $0.5 \mathrm{~mm}$ gaps, more often than not, a microdischarge resulted in a breakdown. With 3 and $5 \mathrm{~ms}$ fronted waves, pbd currents developed in a regular manner. It is found that the current magnitudes increase as front time increases from 1.7 to 3 to $5 \mathrm{~ms}$, with saturation becoming evident towards 5 ms (see Fig. 2).

\section{INFLUENCE OF CONTAMINATION}

Rusted needle cathodes (contaminated condition B) gave the highest pbd currents. These were almost two orders of magnitude higher compared to currents under normal conditions. Condition A resulted in currents that were only about twice the current under normal conditions.

\section{INFLUENCE OF SPARK CONDITIONING}

Spark conditioning of electrodes gives rise to a systematic growth of pbd currents which are also much higher in magnitude. 


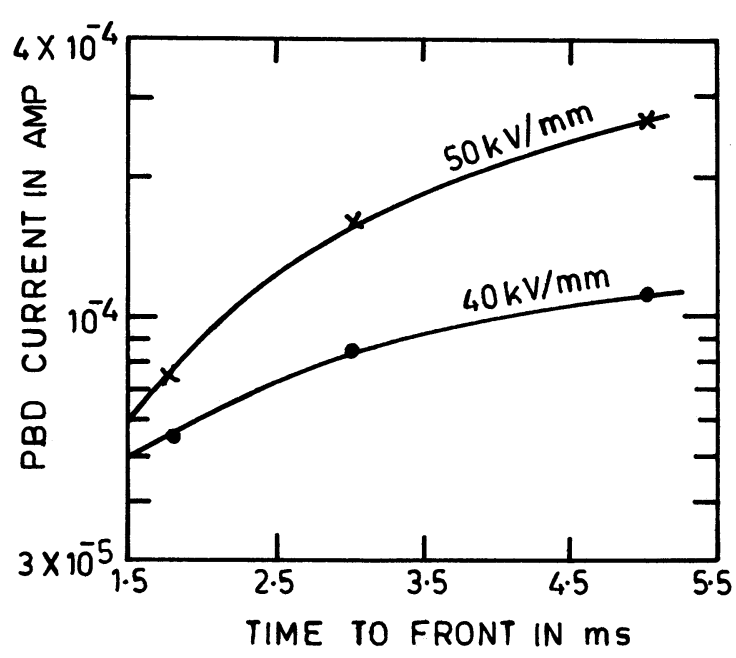

Fig. 2: pbd current as a function of front time of switching impulse excitations for $1.5 \mathrm{~mm}$ gop between $10 \mathrm{~mm}$ diameter hemispherical electrodes

\section{ANALYSIS IN TERMS OF F-N PLOTS}

The voltage-current relationship was analyzed in terms of the classical F-N theory. The F-N plots are shown for various conditions in Figs. 3 and 4 . As may be expected from the asymmetry of the current waveform, the F-N plot is a branched curve (in the clockwise direction) rather than a straight line, (see Fig. 3). In Fig. 3 are also shown F-N plots to illustrate the influence of front time and spark conditioning. Increase of front time from 1.7 to $5 \mathrm{~ms}$ has resulted in higher currents and reduced the values of $\beta$, the field intensification factor. The latter is unexpected and the reasons are also not very clear at present. Spark conditioning may be seen to have shifted the F-N plots upwards very significantly and the curvature of the F-N plots may be seen to have changed. The values of $\beta$ have increased as a result of spark conditioning. These are understandable in the sense that a more rough surface microstructure (due to spark conditioning) is compatible with higher $\beta$ values and hence higher emission currents. Also, such a surface can give off vapors more readily to enable space-charge formation.

\section{DISCUSSION}

Most of the above observations support the view that an anode phenomenon is active. It is believed that the high energy electron beam impinges on the anode causing vaporization. The vapors get ionized and the positive ions, on approaching an emitter sufficiently closely, significantly enhance the emission, explaining the observed high currents. Anode time constants have been reported to be about $1 \mathrm{~ms}$ [12]. If the front time is just comparable or less than the anode time constant, one may expect the anode phenomenon not to be influential. The absence of perceptible pbd currents for front times of $750 \mu \mathrm{s}$ and less and erratic development of pbd currents for $1.7 \mathrm{~ms}$ front impulses appear to be strong evidence of the anode phenomenon being a main cause of the enhanced pbd currents. Further, the slow anode processes would result in a delayed current peak. The longer the time to front, the longer the activity of the anode phenomenon, thereby enhancing emission. Thus higher currents could be expected with larger wave fronts.

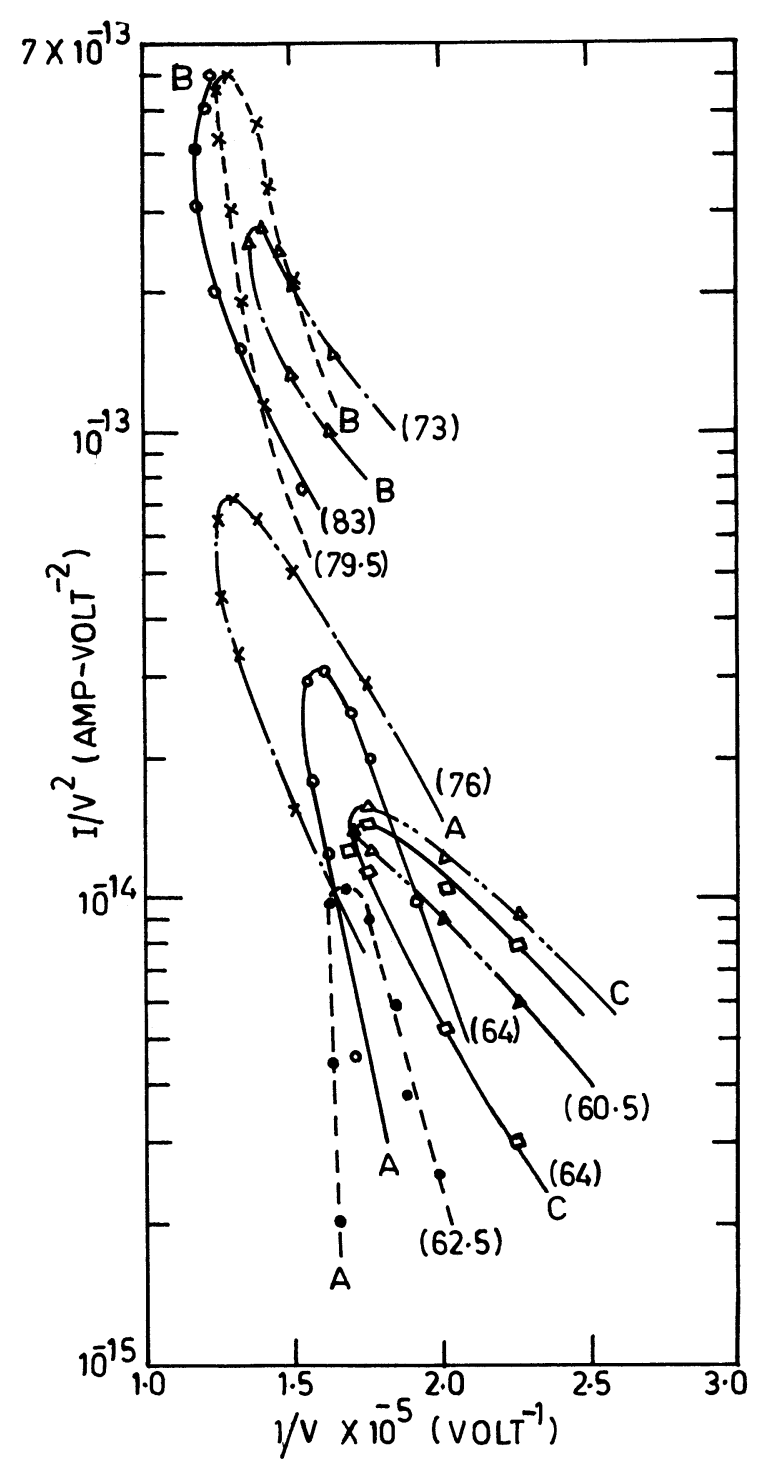

Fig. 3: Typical F-N plots of hemispherical-plane electrodes with $1.5 \mathrm{~mm}$ gap, $A$ and $B$ time to front $=$ $5 \mathrm{~ms}$, A Normal conditioning, B Spark conditioning, $C$ Time to front $=1.7 \mathrm{~ms}$, Normal ac conditioning. All quantities in bracket indicate voltages in $\mathrm{kV}$ (peak).

In view of the above, a midel based on the I-F emission theory of Porotnikov and Rodnevich [13] has been developed that enables computation of pbd currents.

\section{MODEL}

Porotnikov and Rodnevich proposed the following expression for current density considering enhanced emission due to ions (i.e. space charge)

$$
\begin{aligned}
J_{I F}=\frac{1.5 \times 10^{-6} \beta^{2} E^{2}}{\varnothing} \exp \left[\frac{-0.85 \times 10^{7} \phi^{1.5}}{\beta E}+\right. \\
\left.\frac{3 \times \frac{7}{-1 .} \phi^{1.5}}{\beta E}\left(1-\frac{\sqrt{e \beta E}}{\varnothing}\right)\right]
\end{aligned}
$$

Here the usual notation is adopted and as is common, the functions $t^{2}(y)$ and $V(y)$ are equated to unity. Further $\sqrt{e \beta E} / \varnothing<<1$ and is neglected. 


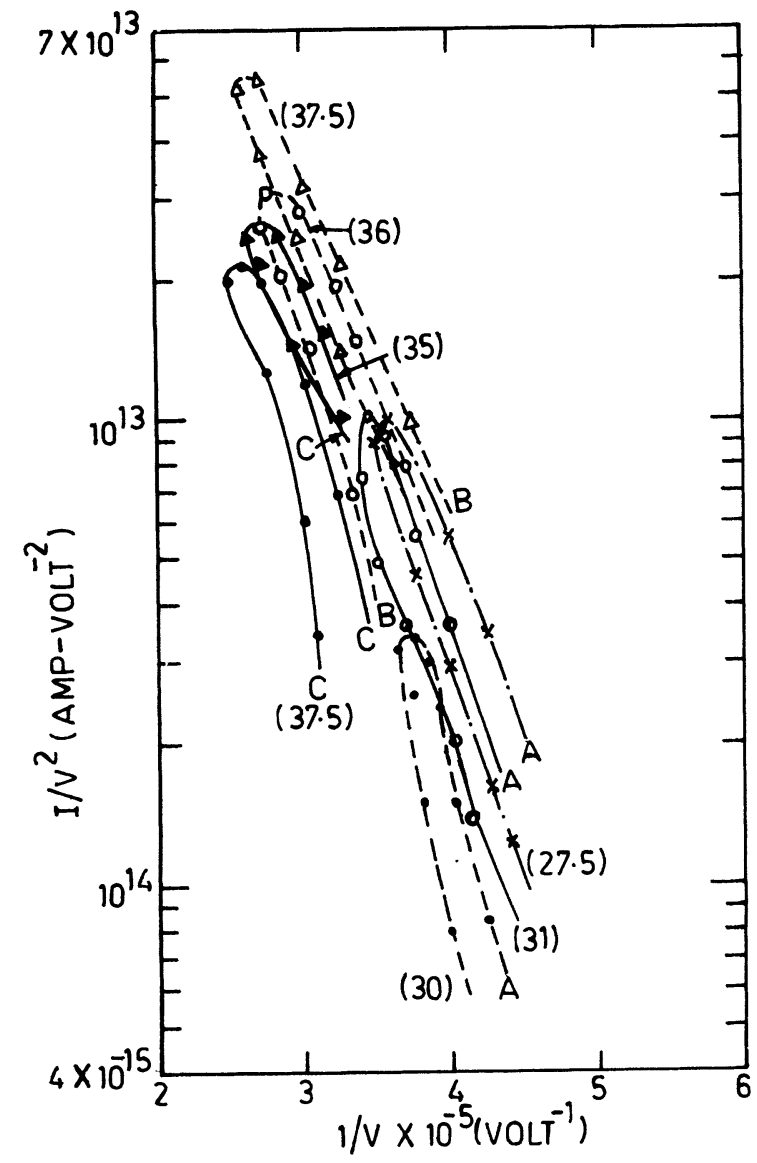

Fig. 4: Typical F-N plots of needle-plane electrodes with $1.5 \mathrm{~mm}$ gap

Front time $=3 \mathrm{~ms}$ (polarity is positive)

A Standard conditions, B Rusted needle, and

C Without liquid nitrogen in trap.

Alz quantities in bracket indicate voltages in $\mathrm{kV}$ (peak).

In Eq. (1) $J I F$ is the current density $\left(\mathrm{A} / \mathrm{cm}^{2}\right), E$ is the average electric field intensity $(\mathrm{V} / \mathrm{cm}), \varnothing$ is the work function (eV) and $\beta$ 'is the field intensification factor. Eq. (1) does not incorporate temporal growth of current but only gives the final stable value. Since, in the present case, the applied voltage is time dependent, it becomes necessary to account for the temporal growth. If the applied field intensity $E_{1}$ is changed to $E_{2}$ in a time intarval $\Delta t$, starting at the instant $t=0$, it is now postulated that current density $J(t)$ for $t>0$ is given by:

$$
J(t)=J_{2}+\left(J_{1}-J_{2}\right) \exp (-t / \alpha)
$$

where $J_{1}$ and $J_{2}$ are current densities as given by Eq. (1), corresponding to $E_{1}$ and $E_{2}$ respectively, and $\alpha$ is the time constant of current growth. Thus even though the voltage is raised instantly to the value $E_{2}$ the current increase occurs gradually with a time constant $\alpha$. Thus in the case of impulses, the current continues to increase for a little while after the voltage has reached the peak. Therefore, the current peak occurs later than the voltage peak. By a similar process it may be seen that the currents will be different for the same instantaneous voltage on the front and tail portions of the wave. A relatively low value $(2 \mathrm{eV})$ of work function and a moderate $\beta$ ralue (200) are assumed for the computation. The F-N curves are plotted for different voltages in $\mathrm{kV}$ (peak). They are shown in Fig. 5. It is seen that experimental and theoretical curves have reasonable similarities, justifying the model.

Apart from the reasonableness of the model discussed above, some discrepancies deserve to be discussed. The work of Latham et a1. [2] and Hurley et al. [3] seem to affirm a "non-metallic" type of emission that are F-N like. The high $\beta$ values of earlier investigations have been attributed by them to field enhancement by the presence of non-metallic particles, rather than microprojections. However, in the present experiments under impulse voltages $\mathrm{F}-\mathrm{N}$ plots have yielded relatively lower $\beta$ values than under dc or ac. Furthermore, "rusted needles" have yielded F-N plots with still lower $\beta$ values, the branches of the plots are much better straight lines and also are closer together. This seems to be in some conflict with the observations of Latham [2] and Hurley [3]. It is also not clear how the waveshape of the applied voltage can influence $\beta$ or if there are any other parameters coming into the picture. Further work is in progress.

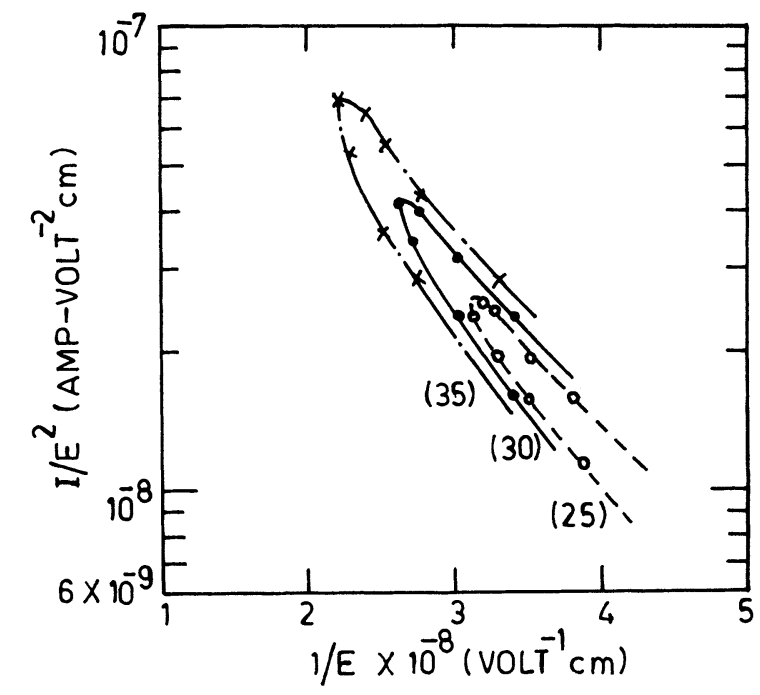

Fig. 5: F-N plots as per the theoretical model Gap $=1.5 \mathrm{~mm}$, Front Time $=3 \mathrm{~ms}, \beta=200, \alpha=0.5 \mathrm{~ms}$, and $\varnothing=2 \mathrm{eV}$

All quantities in bracket indicate voltages in $\mathrm{kV}$ (peak).

\section{CONCLUSIONS}

Prebreakdown currents in vacuum gaps under slow impulse excitations with iront times from 200 us to $5 \mathrm{~ms}$ have been studied. The results strongly indicate that an anode mechanism is at work to influence the current magnitude for front times of $1 \mathrm{~ms}$ or higher. This results in (1) current magnitudes one order higher than under comparable $50 \mathrm{~Hz}$ ac stress, (2) the current peak occurring later than the voltage peak and (3) an "asymmetry" in the current waveform in that the current is higher in the tail for the same voltage. These result in F-N plot: that are branched. It has been possible to explain these on the basis of the ionassisted field emission theory if Porotnikov and Rodnevich after incorporating time dependence in terms of the anode time constant. The agreement is considered satisfactory. Some features of the present 
results, namely, adequacy of lower values of work function and $\beta$ to explain the current magnitude seems to conflict with the "non-metallic" emission model of Latham et al. and Hurley et al.

\section{ACKNOWLEDGMENTS}

The authors wish to thank the authorities of the Indian Institute of Science for supporting this work. One of the authors (K.V.S) is indebted to University Grants Commission, Government of India for sponsoring him under the Quality Improvement Program.

\section{REFERENCES}

[1] R. H. Fowler and L. Nardheim, "Electron Emission in Intense Electric Fields", Proc. Roy. Soc., Vo1. 119A, p 173, (1928).

[2] N. K. Allen, B. M. Cox and R. V. Latham, "The Source of high $\beta$ electron emission sites on broad area high voltage alloy electrodes", J. Phys.D: Appl. Phys., Vol. 12, pp 969-977, (1977).

[3] R. E. Hurley, "Electrical phenomena occurring at the surface of electrically stressed metal cathodes, I Electro luminescence and breakdown phenomena with medium gap spacings", J. Phys.D: App1. Phys., Vo1. 12, pp 2229-2245, (1979).

」 C. C. Erven, J. J. Wavre and R. G. Van Heeswijk, "60 $\mathrm{Hz}$ vacuum breakdown studies in a dynamica1ly pumped system", IV Int. Symp. on Disch. and Elect. Ins. in Vacuum, Waterloo, p 219, (1970).

[5] R. Hackam, "Comparison between $50 \mathrm{~Hz}$ ac and dc vacuum breakdown", J. Phys.D: App1. Phys., Vol. 8, pp L53, (1975).

[6] K.A.N. Kutty and G. R. Nagabhushana, "Prebreakdown current measurements in vacuum gaps stressed by ac voltages", Proc. IEE, Vo1. 123, No. 5, p 475, (1976).
[7] G. Parthasarathy and H. V. Gopalakrishna, "Prebreakdown phenomena in vacuum gaps subject to alternating voltages", Proc. IV Int. Symp. on Disch. and Elect. Insul. in Vacuum, p 214, (1970).

[8] G. A. Farrall, "A study of emitters in broad area electrodes using a phosphor screen", Report No. 71-C068, General Physics Laboratory, Feb (1971).

[9] Bernd-D, Schmidt, "AC breakdown of vacuum gaps at high voltages", III Int. Symp. on High Voltage Engineering, Milan, August (1979).

[10] T. Shiori, I. Ohshima, M. Honda, H. Okumara, H. Takahashi and H. Yoshida, "Impulse voltage field emission characteristics and breakdown dependency upon field strength in vacuum gaps", IEEE Trans., Vo1. PAS-101, No. 10, p 4178, October (1982).

[11] K. Tsuruta, "Prebreakdown field emission current and breakdown mechanism of a small vacuum gap", IEEE Trans., Vo1. EI-18, No. 3, p 204, June (1983).

[12] F. Rohrbach, "Relation between spark time-lag spectra and the mechanisms leading to breakdown between plane Titanium electrodes in Ultra High Vacuum", Proc. IV Int. Symp. on Discharge and Elect. Ins. in Vacuum, Water $100, \mathrm{p} 68$, (1970)

[13] A. A. Porotnikov and B. B. Rodnevich, "I-F Emission", Sov. Phys. Tech. Phys., Vol. 23, No. 6, p 740, (1978).

This paper was presented at the Intermational Symposium on Discharges and Electrical Insulation in Vacuum, Berlin, Germany DDR, 24-28 September 1984.

Manuscript was received 2 May 1985. 